B. ROTATION - CLOSE BINARIES 


\title{
ROTATIONAL INSTABILITIES AND STELLAR EVOLUTION
}

JEAN-PAUL ZAHN

Observatoire de Nice, Le Mont-Gros, 06300 - Nice, France

\begin{abstract}
This review deals with the local instabilities arising when the effects of rotation are taken into account in the evolution of a non-magnetic star.

The Rayleigh and shear instabilities will be examined under the conditions prevailing in radiative zones where the effect of density stratification, thermal diffusion, viscosity and varying chemical composition must be taken into account. The possible consequences on the evolution of a star are finally outlined.
\end{abstract}

In the past decade, many authors have described in detail the evolution of stars of various masses and chemical composition, but the vast majority of the calculated models apply to non-rotating, non-magnetic, and hence spherical stars. To introduce rotation or a magnetic field into the stellar models is not an easy task: the onedimensional problem becomes at least two-dimensional, unless one settles for very drastic simplifications. More seriously, new dependent variables, namely the angular velocity and the components of the magnetic field, enter and one needs additional information, expressed by additional differential equations, about their behaviour in order to determine their distribution inside the star and their evolution with time.

For the sake of simplicity, the effects of rotation and magnetic field are most often studied separately, although one should bear in mind that they may be coupled in many instances. As suggested in the title above, we will deal here only with the instabilities which are triggered by the rotation of a non-magnetic star and which may influence its evolution. We shall narrow our field even further, and focus our attention on the local instabilities, as opposed to the large-scale Eddington-Sweet circulation or to the global instabilities arising when the rotational energy is an appreciable fraction of the total energy of the star. For these latter types of instabilities, we refer the reader to the reviews written by Mestel $(1965,1970)$ and by Ostriker $(1970)$.

As for the local instabilities, the subject has been reviewed in the recent past by several authors, who also discussed their relevance to stellar structure and evolution (Lebowitz, 1967; Strittmatter, 1969; Spiegel and Zahn, 1970; Fricke and Kippenhahn, 1972; see also Ledoux, 1958). For the layman, such reviews may well appear as tiresome enumerations of all presently known instabilities, yet there could hardly be another approach to the problem since no criterion for stability has been found so far. In fact, a closer look at those seemingly impressive lists of local instabilities reveals that all derive from the well-known Rayleigh and shear instabilities, which are present even in an homogeneous incompressible fluid. Our purpose here will be to follow these classical instabilities under the conditions prevailing in the radiative zones of a star, taking into account such effects as density stratification, thermal diffusion, viscosity and varying chemical composition. Having done this, we will conclude with a sketch of the possible consequences of the described rotational instabilities on the evolution of a star. 


\section{Dynamical Instabilities}

Dynamical instabilities occur at some place in the fluid if the equilibrium state is unstable towards an adiabatic inviscid perturbation. Such a dynamical instability has a local character and will settle the fluid into a new state of equilibrium. This new equilibrium state need not be a static one as it may display some motions, turbulent or not. If the equilibrium is dynamical, however, its description cannot be achieved in the adiabatic inviscid frame and one has to take into account both thermal diffusion and viscous transport of momentum.

What all dynamical instabilities have in common is their growth rate: they proceed on a dynamical time-scale which, in the case considered here, will be of the order of a rotation period.

\subsection{AXISYMMETRIC INSTABILITY}

The criterion for axisymmetric instability can be derived in many ways (see for instance Goldreich and Schubert, 1967; Fricke, 1968; James and Kahn, 1970), but in most cases some assumption is made about the spatial behaviour of the perturbation and the criterion obtained in such a way is then no longer rigourously a local one. Here, we prefer to follow the method introduced by Fjörtoft (Eliassen and Kleinschmidt, 1957).

Consider a non-magnetic inviscid star in static equilibrium, whose rotation law $\Omega(\varpi, z)$ depends both on the distance from the rotation axis, $\varpi$, and on the distance from the equatorial plane, $z$. Let us perturb this equilibrium state by a small axisymmetric and purely meridional displacement field $\delta \mathbf{r}(\varpi, z)$ and let us assume that the perturbation proceeds on a time scale short enough to keep it adiabatic. Since the fluid is in an equilibrium state, the first variation $\delta \varepsilon$ of the total (gravitational + internal + kinetic) energy $\varepsilon$ is zero. Its second variation is given by the volume integral

$$
\delta^{2} \varepsilon=\int\left[\delta \mathbf{r} \cdot \mathfrak{M} \cdot \delta \mathbf{r}+\frac{1}{\gamma} \frac{P}{\varrho}\left(\frac{\delta P}{P}\right)^{2}\right] \varrho \mathrm{d} \tau,
$$

where $\delta P$ is the Eulerian pressure perturbation associated with the displacement $\delta \mathbf{r} ; P, \varrho, \gamma$ are the usual notations for pressure, density and the adiabatic exponent.

If the characteristic time of the perturbation exceeds the travel time of a sound wave across the test domain, the second term of this integrand will be negligible compared with the first one, whose sign will determine that of $\delta^{2} \varepsilon$. If the quadratic form $\delta \mathbf{r} \cdot \mathfrak{M} \cdot \delta \mathbf{r}$ is positive definite, the stationary value of $\varepsilon$ is a minimum and the tested equilibrium state is a stable one. On the other hand, if this form is not definite positive, it is possible to choose a virtual displacement $\delta \mathbf{r}$ which will decrease the total energy, thus proving that the equilibrium is then unstable.

This provides a genuine local instability criterion since the virtual displacement $\delta \mathbf{r}$ is entirely arbitrary - apart from the condition that it must vanish on the boundary of the integration domain and that it varies in a time scale which meets the requirements for both adiabaticy and pressure equilibrium to be achieved. 
Now the tensor $\mathfrak{M}$ can be split into two parts, one representing the effect of the density stratification, the other that of the stratification of angular momentum:

$$
\mathfrak{M}=\mathfrak{M}_{1}+\mathfrak{M}_{2}=\frac{1}{C_{p}} \operatorname{grad} S(-\mathbf{g})+\frac{1}{\varpi^{3}} \operatorname{grad}\left(\varpi^{2} \Omega\right)^{2} \operatorname{grad} \varpi
$$

Here $\mathbf{g}$ is the local gravity (including the centrifugal acceleration), and $S$ is the specific entropy:

$$
\frac{1}{C_{p}} \operatorname{grad} S=\frac{1}{\gamma P} \operatorname{grad} P-\frac{1}{\varrho} \operatorname{grad} \varrho=\frac{1}{\varrho} \operatorname{grad}_{\mathrm{AD}} \varrho-\frac{1}{\varrho} \operatorname{grad} \varrho .
$$

(Note also that, when $\mathbf{g}$ and $\operatorname{grad} S$ are colinear or nearly so,

$$
-\mathrm{g} \cdot \frac{1}{C_{P}} \operatorname{grad} S=\frac{|g|}{H_{P}}\left(\nabla_{\mathrm{AD}}-\nabla_{\mathrm{RAD}}\right)
$$

with the usual notations, $H_{P}$ being the pressure scale-height). The discussion of the sign of the two quadratic terms $Q_{1}=\delta \mathbf{r} \cdot \mathfrak{M}_{1} \cdot \delta \mathbf{r}$ and $Q_{2}=\delta \mathbf{r} \cdot \mathfrak{M}_{2} \cdot \delta \mathbf{r}$ leads to a few well known instability criteria which we will review briefly.

If the fluid has a neutral density stratification $(\operatorname{grad} S=0)$, the first quadratic term is identically zero; instability occurs whenever $Q_{2}$ is not positive definite, i.e. when either of the following conditions is met

$$
\frac{\partial}{\partial \varpi}\left(\varpi^{2} \Omega\right)^{2}<0 ; \quad \frac{\partial}{\partial z}\left(\varpi^{2} \Omega\right)^{2} \neq 0 .
$$

The first of those conditions is the Rayleigh criterion for instability in a homogeneous inviscid and non gravitating fluid; the second need not be considered for such a fluid since the rotation law would then violate the Taylor-Proudman theorem and no equilibrium state would be possible (see Greenspan, 1968).

Let us now consider a star with a cylindrical rotation law $\Omega(\varpi)$ and ask if a stable density stratification may prevent the instability when the Rayleigh criterion predicts that it should occur. In such a star, the total body force (gravitational + centrifugal) derives from a potential and the surfaces of constant potential, pressure and density (and hence entropy in a homogeneous star) all coincide; the fluid is said to be barotropic. The quadratic form $Q_{1}$ is positive definite and, except at the exact equator of the star, $Q_{2}$ must also be positive definitive for the total quadratic form $Q_{1}+Q_{2}$ to be of the same character. In other words, if the Rayleigh discriminant is negative, $(\mathrm{d} / \mathrm{d} \varpi)\left(\varpi^{2} \Omega\right)^{2}<0$, some displacements $\delta \mathrm{r}$ will decrease the total energy so that the equilibrium state is proved to be unstable. The unstable displacements are those for which $Q_{1}=0$ or nearly vanishes i.e. those which do not feel the density stratification because they are parallel to the equipotentials.

This result can be extended to more general rotation laws, for which $\Omega$ is a function of both $\varpi$ and $z$. grad $S$ and $\mathbf{g}$ are then no longer colinear and the angle between them is determined by the baroclinic condition

$$
\frac{1}{C_{P}} \operatorname{grad} S \times(-\mathbf{g})+\frac{1}{\varpi^{3}} \operatorname{grad}\left(\varpi^{2} \Omega\right)^{2} \times \operatorname{grad} \varpi=0
$$


which is obtained by taking the curl of the equation of motion. The necessary and sufficient condition for the total quadratic form $Q_{1}+Q_{2}$ to be positive definite is

$$
\left[\frac{1}{C_{P}} \operatorname{grad} S \times \frac{1}{\varpi^{3}} \operatorname{grad}\left(\varpi^{2} \Omega\right)^{2}\right] \cdot[-\operatorname{g} \times \operatorname{grad} \varpi]>0 \text {. }
$$

If this condition is violated, one can always choose a virtual displacement in such a way that it will decrease the total energy. The configuration is thus unstable and this is known as the baroclinic instability. The vector condition (5), together with (4), shows that this instability arises whenever the specific angular momentum $\varpi^{2} \Omega$ decreases towards the equator on a surface of constant specific entropy. This criterion is more general than the Rayleigh criterion, which, of course, it includes. It shows also that a stable density stratification permits certain rotation laws which depend on the $z$ coordinate, thereby violating the Taylor-Proudman theorem.

\subsection{Shear INSTABility}

Unfortunately, it has not yet been possible to extend the stability criterion given above to more general, non-axisymmetric, virtual displacements. However, the instability which is likely to be most prominent in a fluid which is not in solid body rotation when perturbed by non-axisymmetric disturbances, is the so-called shear instability.

In a plane parallel shear flow, a sufficient condition for stability is that the velocity profile present no inflection point. This theorem has also been established by Rayleigh, and its counterpart for a cylindrical flow is that the expression

$$
\frac{\mathrm{d}}{\mathrm{d} \varpi}\left[\frac{1}{\varpi} \frac{\mathrm{d}}{\mathrm{d} \varpi}\left(\varpi^{2} \Omega\right)\right]
$$

does not change sign in the domain considered. What happens when this expression vanishes is not clear, although it is likely that dynamical instability will appear for a large variety of velocity profiles. But this is of rather academic interest since laboratory experiments and some theoretical investigations have shown that the viscosity is able to destabilize any flow, even if it is claimed to be stable according to Rayleigh's theorem above, provided that the Reynolds number $V L / v$ exceeds some critical value which is of order $10^{3}$. ( $V$ and $L$ are respectively typical values for the velocity and the dimension of the domain and $v$ is the kinematic viscosity).

Here again the flow may be stabilized by a (stable) density stratification, provided that the Richardson number $\boldsymbol{R}_{i}$ exceeds some critical value:

$$
R_{i}=\frac{g}{C_{P}} \frac{\mathrm{d} S}{\mathrm{~d} z} /\left(\frac{\mathrm{d} v}{\mathrm{~d} z}\right)^{2}>\frac{1}{4}
$$

A similar condition probably applies also to differential rotation, the velocity gradient being replaced by $\varpi \operatorname{grad} \Omega$, and only the components of $\mathbf{g}$ and $\operatorname{grad} S$ parallel to the gradient of $\Omega$ being retained in the criterion:

$$
\cos ^{2} \alpha \frac{g}{C_{P}} \frac{\mathrm{d} S}{\mathrm{~d} r}>\frac{1}{4}(\varpi \operatorname{grad} \Omega)^{2}
$$

(here $\alpha$ is the angle between $\mathrm{g}$ and $\operatorname{grad} \Omega$ ). 


\section{Destabilization Through Thermal Diffusion}

As we have just seen, a suitable density stratification can stabilize flows which would otherwise be unstable. But this situation changes drastically, at least in a homogeneous fluid, if the perturbations are not constrained to be adiabatic. The reason for this is that thermal diffusion smoothes out the temperature fluctuations, and hence the density perturbations since on the time scale considered here the medium is in almost perfect pressure equilibrium. As a result, the stabilizing buoyancy forces are weakened and, in some cases, can no longer prevent the muted instability.

\subsection{AxisYmmetric InStabiLITy}

In a rotating star, the effect of smoothing the density perturbation is to decrease the quadratic term $Q_{1}$ in $\delta^{2} \varepsilon$ (Equation (1)) to a point where it becomes negligible compared to $Q_{2}$. Instability then sets in whenever $Q_{2}$ fails to be a definite positive quadratic form, and this happens, as we have already seen, when

$$
\frac{\partial}{\partial \varpi}\left(\varpi^{2} \Omega\right)^{2}<0 \text { or } \frac{\partial}{\partial z}\left(\varpi^{2} \Omega\right)^{2} \neq 0 .
$$

Goldreich and Schubert (1967) and Fricke (1968) were the first to describe this effect in the astrophysical context, but credit should also be given to Yih (1961) for having called attention to the destabilizing role of thermal conduction in a cylindrically rotating flow. The question of the growth rate of this instability has not yet been settled, although in the linear phase of its development it is tied to the thermal time scale. James and Kahn (1970) endeavoured to follow the perturbation into the nonlinear regime, where its behaviour must bear much resemblance with that of thermohaline convection.

In thermohaline convection, an unstable salt gradient is stabilized by a stable temperature - (and thus density) - gradient. As Goldreich and Schubert pointed out, the situation is very similar to that of a rotating fluid of low Prandtl number* since, in both cases, the thermal diffusivity is much larger that either the molecular diffusivity of salt or the viscous diffusivity of angular momentum.

For a comprehensive account of thermohaline convection, we refer to Spiegel(1972). Laboratory experiments and numerical investigations show that the dominant unstable modes are of high horizontal wave number; they evolve in what is called the salt fingers. As those fingers reach an appreciable vertical size, they seem to become collectively unstable and turn into layers with discontinuities in concentration, such as those observed in the oceans. Finally, it is found that such layering can be destroyed by sufficiently strong turbulence.

It is very likely that the Goldreich-Schubert-Fricke instability will also result in a similar layering of angular momentum, which in turn, will then become eligible for shear instability, if it has not been so before.

* The Prandtl number is the ratio of the kinematic viscosity to the thermal diffusivity $\sigma=v / \kappa$. 


\subsection{SHEAR INSTABILITY}

Such a breakdown of the density stabilization is also expected in the shear flow. In the Earth's atmosphere, turbulent motions are observed in regions for which the Richardson criterion predicts strong stability. In order to explain this, Townsend (1958) took thermal diffusion into account in the energy balance of a turbulent shear flow. He showed that this effect lowers considerably the critical Richardson number if the radiative cooling time of the perturbations is small enough, so that

$$
t_{\text {cool }}\left|\frac{\mathrm{d} v}{\mathrm{~d} z}\right|<\sim \frac{1}{6}
$$

Townsend introduces then a new non-dimensional number, the flux Richardson number, which must be larger than unity for the turbulence to be prevented by the density stratification:

$$
\frac{g}{C_{P}} \frac{\mathrm{d} S}{\mathrm{~d} z} t_{\text {cool }} /\left|\frac{\mathrm{d} v}{\mathrm{~d} z}\right|>\sim 1 .
$$

Moore and Spiegel (1964) have argued that one should take for $t_{\text {cool }}$, the cooling time corresponding to the smallest scale which is optically thick in the medium. This length is of the order of a centimeter in stellar interiors and the application of Townsend's criterion would then lead to critical rotation laws with exceedingly small gradients.

In Townsend's treatment, the cooling time is that associated with the integer scale of the turbulence, which is probably much larger than the one centimeter above. Unfortunately, in the absence of direct observations one has to resort to some guesswork about it. It seems however reasonable to take for it the shortest length $l$ whose Reynolds number still remains supercritical:

$$
\frac{l^{2}}{v}\left|\frac{\mathrm{d} v}{\mathrm{~d} z}\right|=R_{\mathrm{crit}}
$$

where $R_{\text {crit }}$ is the critical Reynolds number $\left(\sim 10^{3}\right)$ for the given velocity profile. Among all the scales present in the spectrum of the turbulence, this one is likely to stand out: it has the largest growth-rate on the source side of the spectrum, where energy is fed into the turbulence from the unstable laminar flow.

Using the radiative cooling time corresponding to this scale, one finds that the critical Richardson number is increased to a value of the order $\left(\sigma R_{\text {crit }}\right)^{-1}$ (see footnote on p. 189), typically $10^{3}$ or $10^{4}$ in stellar interiors. This leads to the following estimation of the gradient of angular velocity which cannot be stabilized by a given density stratification:

$$
(\varpi \operatorname{grad} \Omega)^{2}>\left(\sigma R_{\text {crit }}\right) \cos ^{2} \alpha \frac{g}{C_{p}} \frac{\mathrm{d} S}{\mathrm{~d} r}
$$


or

$$
(\varpi \operatorname{grad} \log \Omega)^{2}>\left(\sigma R_{\mathrm{crit}}\right) \cos ^{2} \alpha\left(\frac{g}{\Omega^{2} H_{p}}\right)\left(\nabla_{\mathrm{AD}}-\nabla_{\mathrm{RAD}}\right)
$$

with the usual notations.

A perturbation of the scale assumed above has a growth time of order $t=l^{2} / v=$ $=R_{\text {crit }}|\varpi \operatorname{grad} \Omega|^{-1}$, only three or four orders of magnitude larger than the dynamical time scale. We may thus conclude that gradients of angular velocity which are larger than those indicated by condition (10) will be wiped out in time which is short compared to any time characterizing the thermal or nuclear evolution of the star.

\section{Stabilization Through Chemical Composition Gradients}

We have just seen how efficiently thermal diffusion suppresses the stabilizing effect of a density stratification. But this is true only as long as the fluid is homogeneous. If the density gradient is sustained by a stable gradient of chemical composition, the density fluctuations cannot be cancelled completely by thermal diffusion and the remaining buoyancy forces may still be strong enough to prevent the instability.

\subsection{AXISYMMETRIC INSTABILITY}

In the case of axisymmetric disturbances, this can be seen by deriving the corresponding second variation $\delta^{2} \varepsilon$ of the total energy, as in the case of adiabatic perturbations. The star is assumed to be in overall secular stability, so that the first variation $\delta \varepsilon$ is again zero. One has to assume also that the perturbation evolves slowly enough so that the Eulerian variations of pressure and temperature can be neglected. The variation of density is then due only to the advection of matter of varying molecular weights

$$
\delta \varrho / \varrho=-\delta \mathbf{r} \cdot \operatorname{grad} \log \mu
$$

and the quadratic term $Q_{1}$ becomes:

$$
Q_{1}=\delta \mathbf{r} \cdot(-\operatorname{grad} \log \mu)(-\mathbf{g}) \cdot \delta r .
$$

It is not possible to derive a general secular stability criterion, as was done previously for the dynamical stability, since the four vectors involved in the tensor $\mathfrak{M}$ are no longer related by an equation similar to the baroclinic one (4). (For a perfect gas, $\operatorname{grad} \log \mu / T$ is still related to the three other vectors, but $\operatorname{grad} \log \mu$ is not). However, one can proceed along the following lines.

Since the stabilization comes from the quadratic term $Q_{1}$, let us take the most favorable case, for which the vectors $\mathrm{g}$ and $\operatorname{grad} \log \mu$ are colinear: $Q_{1}$ is then positive definite, i.e. positive except for $\boldsymbol{\delta} \mathbf{r}$ perpendicular to those vectors where it vanishes. In this case, it is easy to show that the total quadratic form $\delta \mathbf{r} \cdot \mathfrak{M} \cdot \delta \mathbf{r}$ is definite positive if and only if:

$$
\left(\frac{1}{\varpi^{3}} \operatorname{grad}\left(\varpi^{2} \Omega\right)^{2} \times \operatorname{grad} \varpi\right)^{2}
$$




$$
<4\left(-|g| \frac{\mathrm{d}}{\mathrm{d} \zeta} \log \mu\right)\left(1_{\zeta} \times \frac{1}{\varpi^{3}} \operatorname{grad}\left(\varpi^{2} \Omega\right)^{2}\right) \cdot\left(1_{\zeta} \times \operatorname{grad} \varpi\right)
$$

where $\mathbf{1}_{\zeta}$ is the unit vector in the vertical direction, i.e. $\mathbf{1}_{\zeta}=-\mathbf{g} /|g|$. If the angular velocity is a function of the vertical coordinate $\zeta$ only, or if the horizontal variation of $\Omega$ can be neglected compared to the vertical variation, this awkward condition takes a much simpler form:

$$
-|g| \frac{\mathrm{d}}{\mathrm{d} \zeta} \log \mu>\frac{1}{4}\left(\varpi \frac{\mathrm{d} \Omega}{\mathrm{d} \zeta}\right)^{2} .
$$

One may therefore conclude, as did Goldreich and Schubert, that a sufficiently strong gradient of molecular weight is capable of suppressing the instability named after these authors.

\subsection{SheAR INSTABILITY}

Such a $\mu$-gradient will also inhibit the shear instability; if, in the derivation of the Richardson criterion, one assumes the perturbations to be isothermal instead of adiabatic, the gradient of molecular weight takes the place of the density gradient. Transposed to a rotating fluid, the Richardson criterion for stability then becomes :

$$
-\cos ^{2} \alpha|g| \frac{\mathrm{d}}{\mathrm{d} \zeta} \log \mu>\frac{1}{4}(\varpi \operatorname{grad} \Omega)^{2}
$$

which is identical to condition (13) above, but with no restrictive assumption about the rotation law ( $\alpha$ is again the angle between $\operatorname{grad} \Omega$ and $\mathbf{g}$; the $\mu$-gradient is supposed to be purely vertical).

\section{The Evolution of Rotating Stars}

Few attempts have been made so far to follow the detailed history of a rotating star (see e.g., Kippenhahn et al., 1970), although it has been known at least since Eddington's work that rotation can play an important role in the evolution of a star. True, it is only very recently that the destabilizing role of thermal diffusion has been recognized and this explains why most evolutionary calculations take the rotational instabilities into account only very crudely, and why some neglect them completely (e.g. Sakurai, 1972).

In the absence of more detailed calculations, one can hardly do better than sketch roughly what, with some likelihood, appears to be the evolution of a rotating star.

If the star, when it first reaches the main sequence, is homogeneous as the result of convective mixing during the Hayashi phase, it will settle into a cylindrical rotation state due to the interplay between the two instabilities described above (GoldreichSchubert-Fricke's and shear). This adjustment takes only a very short time, of the order of $10^{3}$ or $10^{4}$ rotation periods.

In the case of a late-type star, the rotation law is then entirely determined by the 
boundary conditions at the bottom of the outer convection zone*. If, as it is now believed after Schatzman (1962), the rotation of the convection zone is slowed down by a stellar wind sustained by strong magnetic fields, the rotation of the radiative interior will adjust to it in the short time already mentioned. It is only after this braking mechanism has become very weak, probably when its (negative) $e$-folding time has become comparable with the nuclear evolution time, that the motions in the radiative core of the star have damped sufficiently so that a gradient of molecular weight can be established. After this time, the star evolves inhomogeneously and its core is shielded from further rotational instability. After the main-sequence phase, the contracting core will conserve its angular momentum and will spin much faster than the expanding envelope.

In this scheme, the present Sun would have a core rotating with the angular velocity determined by the angular momentum it had at the beginning of its inhomogeneous phase. Since the Sun has probably not slowed down subsequently by more than a factor of two or three, it is not likely that it possesses the fast spinning core needed to support Dicke's theory (1964), or to lower the calculated neutrino flux to the presently observed level (see Demarque's contribution at this symposium).

In an early type star, the rotation law $\Omega(\varpi)$ is similarly determined by the convective core, but only inside the cylinder tangent to it at its equator. Outside this region, the situation is more complex; it is probably the Eddington-Sweet circulation which plays the main role in redistributing angular momentum, within the limit fixed by condition (10) which does not tolerate departures from solid rotation much larger than a few per cent. The gradients of molecular weight built by this circulation (Mestel's $\mu$-barriers, 1953) and those later left behind by the receding convective core are probably strong enough to shield the central regions from the rotational instabilities as in the case of a low mass star.

All this has clearly to be checked by detailed evolutionary calculations. The only excuse for the present speculations is that they may stimulate such much needed investigations.

\section{Acknowledgements}

I wish to thank Prof. R. Van der Borght and his colleagues of Monash University, where this review has been prepared, for their generous hospitality. I am also indebted to Prof. R. H. Koch for having critically read the manuscript and greatly improved the English.

\section{References}

Dicke, R. H.: 1964, Nature 202, 432.

Eliassen, A. and Kleinschmidt, E. : 1957, Handbuch der Physik 48, 64.

\footnotetext{
* Should the differential rotation imposed by the convection zone be strong enough to trigger the shear instability according to condition (10), the angular velocity of the radiative interior would probably remain in neutral equilibrium and connect with the angular velocity in the convection zone through a turbulent Ekman layer.
} 
Fricke, K. J.: 1968, Z. Astrophys. 68, 317.

Fricke, K. J. and Kippenhahn, R.: 1972, Ann. Rev. Astron. Astrophys. 10, 45.

Goldreich, P. and Schubert, G.: 1967, Astrophys. J. 150, 571.

Greenspan, H. P.: 1968, in G. K. Batchelor and J. W. Miles (ed.), The Theory of Rotating Fluids, Cambridge University Press, London, p. 2.

Howard, L. N., Moore, D. W., and Spiegel, E. A.: 1967, Nature 214, 1297.

James, R. A. and Kahn, F. D.: 1970, Astron. Astrophys. 5, 232.

Kippenhahn, R., Meyer-Hofmeister, E., and Thomas, H. C. : 1970, Astron. Astrophys. $5,155$.

Lebovitz, N. R.: 1967, Ann. Rev. Astron. Astrophys. 5, 465.

Ledoux, P.: 1958, Handbuch der Physik 51, 605.

Mestel, L.: 1953, Monthly Notices Roy. Astron. Soc. 113, 716.

Mestel, L.: 1965, Stars and Stellar Systems 8, 465.

Mestel, L. : 1970, Circular Letter No. 10, IAU Comm. 35.

Moore, D. W. and Spiegel, E. A.: 1964, Astrophys. J. 139, 48.

Ostriker, J. P.: 1970, in A. Slettebak (ed.), 'Stellar Rotation', IAU Colloq. 4, 147.

Sakurai, T.: 1972, Publ. Astron. Soc. Japan 24, 153.

Schatzman, E.: 1962, Ann. Astrophys. 25, 18.

Spiegel, E. A.: 1972, Ann. Rev. Astron. Astrophys. 10, 261.

Spiegel, E. A. and Zahn, J. P.: 1970, Comments Astrophys. Space Phys. 2, 178.

Strittmatter, P. A.: 1969, Ann. Rev. Astron. Astrophys. 7, 665.

Townsend, A. A. : 1958, J. Fluid Mech. 4, 361.

Yih, C.-S. : 1961, Phys. Fluids 4, 806.

\section{DISCUSSION}

(Note: Several questions have been raised in the discussion by Drs Demarque, Schatzman and Tayler concerning the rotational evolution of the Sun. Dr Zahn has preferred to include his answers in the paper itself, whose last paragraph has been rewritten for this purpose.)

Tayler: Could I make a comment on a problem that Dr Zahn has not actually spoken about. That is the interference of rotation with convection, which of course is buried in his criteria somewhere. If one wants a simple sentence to bear on this, rotation will interfere with convection, if the rotation time is short compared to the lifetime of the convective element.

Zahn: According to the criterion (2) you have always some displacement which should be unstable, even if you have a stabilizing rotation.

Tayler: There will always be some unstable perturbation. I said it will interfere with convection, I did not say it would suppress it.

Zahn: Yes.

Rodgers: It seems to me that the only objects I can think of straight away which are regarded as purely radial pulsators and which may have relatively high observed rotational velocities are the stars which get into the instability zone through the McCray mechanism, as blue stragglers. We are talking, if we want to be specific, about one star called HD 6870, where we have $V \sin i$ typically around $120 \mathrm{~km}$ per second. Prof. Eggen has observed it as a low amplitude variable of the order of a few hundredths of a magnitude but with typical $\delta$ Scuti characteristics. What I want to know is what do you say about the effects of rotation on radial pulsation?

Zahn: This was a part of Prof. Ledoux's topic. As he has mentioned the radial oscillation will also be affected by rotation, there will be a shift in frequency, a slight one, but that is all.

Ledoux: It is difficult to define a purely radial oscillation in something that is rotating pretty fast, but you still have some kind of pseudo radial mode which is usually not too different in period from the radial mode.

Zahn: But the blue stragglers raise another question. How do they occur at all? You remember that Prof. Mestel found that the $\mu$-gradients will prevent mixing in the star. It may well be that if there is a stronger rotation present those $\mu$-gradients will be broken or something like that happens. Has someone looked in detail at that?

Cox: Let me ask the same question as Dr Rodgers. Is it true that rotation will decrease the amplitude of purely radial pulsation?

Zahn: To tell something about the amplitude you have to do nonlinear calculations. The amplitude 
may be reduced because the Coriolis force will try to make the oscillation more horizontal. But you would have to look in detail, I have no real answer.

Vardya: This is similar to what Dr Demarque asked earlier. A $\mu$-gradient will be built up also as you go to the core because of ionisation. How much of a $\mu$-gradient do you want? This will occur when the star is homogeneous.

Zahn: The $\mu$-gradient which enters in the instability criteria (13) and (14) is that caused by a genuine variation of chemical composition. The state of ionisation adjusts too fast to a change in pressure or temperature to play here any role. Moreover, the resulting $\mu$-gradient would be destabilizing $(\mu$-increasing outwards). 